\title{
AN APPLICATION OF THE GENERALIZED NORM RESIDUE SYMBOL
}

\section{KATSUHIKO MASUDA}

Let $k$ be an algebraic number field of finite degree or an algebraic function field of dimension 1 with finite constant field and let $K / k$ be a finite, separably Galois extension field. We call an algebraic extension field $L$, not necessarily Galois nor necessarily of finite degree, of $K$ an everywhere locally abelian extension, denoted in the following as EL-abelian extension, for brevity, of $K$ over $k$, if and only if for every finite extension $L^{\prime}$ of $K$ in $L$ and every valuation $v$ of $L$ it holds that the completion field $L_{v}^{\prime}$ of $L^{\prime}$ with reference to $v$ is a composition field of $K_{v}$ and an abelian extension $M_{v}$ of $k_{v}$ in $L_{v}^{\prime}$, where we denote by $k_{v}$ and $K_{v}$ the closures of $k$ and $K$ in $L_{v}^{\prime}$, respectively.

Let $\Omega$ be an algebraic closure of $K, B_{K / k}$ the maximum separably EL-abelian extension of $K / k$ in $\Omega, C_{K / k}$ the maximum separably central extension of $K / k$ in $\Omega$ (i.e. the maximum extension $C_{K / k}$ which is separably normal over $k$, contains $K$, and has the Galois group of $C_{K / k} / K$ in the center of the Galois group of $\left.C_{K / k} / k\right), I_{k}$ and $I_{K}$ the groups of the idèles of $k$ and $K, A_{k}$ and $A_{K}$ the maximum separably abelian extensions of $k$ and $K$ in $\Omega$, respectively. Let $D_{K / k}=B_{K / k}$ $\cap C_{K / k}$. Then, the norm residue symbol $\sigma_{k}$ in $A_{k} / k$ is, if restricted into $N_{K / k} I_{K}$, extensible into a homomorphism $\sigma_{K / k}$ of $N_{K / k} I_{K}$ into the Galois group $G\left(D_{K / k} / K\right)$. We shall study in the present article arithmetical meanings of the extensibility.

In $\S 1$, we shall define $\sigma_{K / k}$ precisely, in $\S 2$, study the kernel and obtain a principal genus theorem (Theorem 2), from which will follow easily a certain generalized formulation of some fundamental theorems in the class field theory. ${ }^{1}$ Combining it with a known property ${ }^{2}$ of the quotient group of the connected component of the unit element of the idèle class group by the natural image of the maximal compact subgroup of the connected component of the unit element of the idèle group, we shall obtain, in $\$ 3$, our main result (Theorem 8 ), which concerns total norm residues in the principal idèle group of $k$ for $K / k$, and which is, the author thinks, an idèle-theoretic reconstruction of a research of Scholz. ${ }^{3}$

\footnotetext{
${ }^{1}$ Cf. $[1 ; 2 ; 4]$.

2 Cf. [4].

${ }^{8} \mathrm{Cf} .[3]$.
}

Received by the editors July 25,1958 . 
1. Definition of $\sigma_{K / k}$. Let $k, K, \Omega, B_{K / k}, C_{K / k}, D_{K / k}, I_{k}, I_{K}, A_{k}, A_{K}$, and $\sigma_{k}$ be, throughout the present article, as stated above. Let $M$ be the set of the prime divisors of $k, k_{p}, k_{p}^{*}$ and $\Omega_{p}$ with $p$ in $M$ the completion field of $k$ for $p$, the multiplicative group of the nonzero elements of $k_{p}$ and an algebraic closure, not complete, of $k_{p}$, respectively. Let $V$ be the set of the normalized valuations of $\Omega, p$ with $p$ in $M$ the set of the normalized valuations of $\Omega$ corresponding, if restricted into $k$, to $p, N$ a complete system of representatives of the classification of $V$ as the set-theoretic direct sum of $\bar{p}$ with $p$ in $M$. Clearly, there exists a canonical one-to-one correspondence $\iota$ of the set $\bar{p}$ and the set [p] of the isomorphisms of $\Omega / k$ into $\Omega_{p} / k$, we denote by $v^{*}$ with $v$ in $V$ the isomorphism $\iota(v)$ of $\Omega$ into $\Omega_{p}$, and let $K_{p}$ $=k_{p} v^{*}(K)$ with $v$ in $\not \cap N$ and $K_{p}^{*}$ the multiplicative group of the nonzero elements in $K_{p}, A_{p}$ the maximum abelian extension of $k_{p}$ in $\Omega_{p}$, and $\sigma_{p}$ the norm residue symbol in $A_{p} / k_{p}$. As is well known, $\sigma_{p}$ gives canonically an isomorphism $\sigma_{p}^{\prime}$ of $N_{K_{\mathfrak{p}} / k_{p}} K_{p}^{*}$ into the Galois group $G\left(K_{p} A_{p} / K_{p}\right)$ and we define for $v$ in $p \cap N$ an isomorphism $\sigma_{v}$ of $N_{K_{p} / k_{p}} K_{p}^{*}$ into $G\left(D_{K / k} / K\right)$ such that it holds for every $\alpha$ in $D_{K / k}$ and every a in $N_{K_{p} / k_{p}} K_{p}^{*}$

$$
v^{*}\left(\sigma_{v \bar{p}}(\mathfrak{a}) \alpha\right)=\sigma_{p}^{\prime}(\mathfrak{a}) v^{*}(\alpha){ }^{4}
$$

As, from the supposition, $G\left(D_{K / k} / K\right)$ lies in the center of $G\left(D_{K / k} / k\right)$, $\sigma_{v}$ is independent from the choice of $v$ in $\bar{p}$, and we denote it, changing the notation, by $\sigma_{\bar{p}}$. Then, it holds clearly for every $\mathfrak{A}$ in $K_{P}^{*}\left(\right.$ in $I_{K}$ )

$$
\sigma_{K}^{\prime}(\mathfrak{A})=\sigma_{p}\left(N_{K P / k_{p}} \mathfrak{U}\right),
$$

where we denote by $\sigma_{K}^{\prime}$ the norm residue symbol in $D_{K / k} / K$, by $P$ a prime divisor of $p$ in $K$, by $K_{P}^{*}$ the multiplicative group of the nonzero elements in the completion field $K_{P}$ of $K$ for $P$. Let $I_{\boldsymbol{k}}^{*}$ be the restricted direct product of $k_{p}^{*}$ with $p$ in $M$, contained in $I_{k}$, and we define a mapping $\sigma$ of $I_{\boldsymbol{k}}^{*} \cap N_{K / k} I_{K}$ in to $G\left(D_{K / k} / K\right)$ by

$$
\sigma\left(\mathfrak{a}_{p_{1}} \mathfrak{a}_{p_{2}} \cdots \mathfrak{a}_{p_{\boldsymbol{s}}}\right)=\sigma_{\bar{p}_{1}}\left(\mathfrak{a}_{p_{1}}\right) \sigma_{\bar{p}_{2}}\left(\mathfrak{a}_{p_{2}}\right) \cdots \sigma_{\bar{p}_{\boldsymbol{s}}}\left(\mathfrak{a}_{p_{\boldsymbol{s}}}\right)
$$

for $\mathfrak{a}_{p_{i}}$ in $N_{K_{p_{i}} / k_{i}} K_{p_{i}}^{*}$. Clearly, $\sigma$ is a homomorphism and $\sigma\left(I_{\boldsymbol{k}}^{*} \cap N_{K / k} I_{K}\right)$ is dense in $G\left(D_{K / k} / K\right)$. Let $\mathfrak{a}=\left(\mathfrak{a}_{p}\right)$ be an idèle in $N_{K / k} I_{K}$. We can take an idèle $\mathfrak{B}=\left(\mathfrak{B}_{P}\right)$ in $I_{K}$ such that $\mathfrak{a}=N_{K / k} \mathfrak{B}$ and $\mathfrak{B}_{Q}=1$ for every prime divisor $Q$ of $K$ not corresponding to any of the restrictions into $K$ of the valuations in $N$. Then, it follows from (2) the following formal equality

$$
\prod_{P} \sigma_{K}^{\prime}\left(\mathfrak{B}_{P}\right)=\prod_{p} \sigma_{\bar{p}}\left(\mathfrak{a}_{p}\right)
$$

${ }^{4}$ Cf. [4]. 
where $P$ and $p$ run over all prime divisors of $K$ and of $k$, respectively. As the left product has a limit in $G\left(D_{K / k} / K\right)$, the right one has the same limit and we extend continuously into a homomorphism $\sigma_{K / k}$ of $N_{K / k} I_{K}$ into $G\left(D_{K / k} / K\right)$, defining for $\mathfrak{a}=\left(\mathfrak{a}_{p}\right)$ in $N_{K / k} I_{K} \sigma_{K / k}(\mathfrak{a})$ as

$$
\sigma_{K / k}(\mathfrak{a})=\prod_{\boldsymbol{p}} \sigma_{\bar{p}}\left(\mathfrak{a}_{p}\right)
$$

Evidently, it follows from (2) and (5) the following fundamental equality, which we state as

THEOREM 1.

$$
\sigma_{K}^{\prime}(\mathfrak{H})=\sigma_{K / k}\left(N_{K / k} \mathfrak{H}\right)
$$

( $\left.\mathfrak{A} \in I_{K}\right)$.

2. The kernel of $\sigma_{K / k}$. Let $L$ be a finite separably algebraic extension of $k$ in $\Omega$, Galois over $k$ and abelian over $K$. Let $A, B, C$, and $D$ be $A_{k} \cap L, B_{K / k} \cap L, C_{K / k} \cap L$, and $D_{K / k} \cap L$, respectively, and $I_{L}$, $\sigma_{K / k}^{D}, \sigma_{K}^{L}$ be the group of the idèles of $L$, the homomorphism of $N_{K / k} I_{K}$ into the Galois group $G(D / K)$ obtained canonically from $\sigma_{K / k}$, and the norm residue symbol in $L / K$, respectively. Let $H(L / K)$ and $F$ be the inverse images in $I_{K}$ by $\sigma_{K}^{L}$ of $G(L / L)$ and $G(L / D)$, respectively, and $F^{*}$ be the inverse image in $I_{K}$ by $N_{K / k}$ of $N_{K / k} H(L / K)$. Then, there exists for $\mathbb{C}$ in $F^{*}$ an idèle $\mathfrak{S}$ in $H(L / K)$ such that $N_{K / k} \mathfrak{E}$ $=N_{K / k} \mathfrak{S}$ and we obtain from Theorem 1

$$
\stackrel{D}{\sigma_{K}}(\mathfrak{S})=\stackrel{D}{\sigma_{K / k}}\left(N_{K / k} \mathfrak{S}\right)=\stackrel{D}{\sigma_{K / k}}\left(N_{K / k} \mathfrak{W}\right)=\stackrel{D}{\sigma_{K}}(\mathfrak{S})=1,
$$

where we denote by $\sigma_{K}^{D}$ the norm residue symbol in $D / K$. Thus,

$$
F^{*} \subset F \text {. }
$$

As $F^{*} \supset H(L / K)$ and, from the supposition, $L$ is of finite degree over $K$, there exists a subfield $L^{\prime}$ of $L$, corresponding to $F^{*}$. Let $P$ for $p$ in $M$ be the prime divisor of $K$, corresponding to the restriction into $K$ of $v$ in $\not \cap N$, and $I_{K, P}$ the subgroup of $I_{K}$ consisting of the idèles with 1 as the component everywhere except at $P$. We shall identify in the following, without contradiction, $I_{K, P}$ and $K_{p}^{*}$, when we shall deal with local properties at $p$, so it holds for $\mathfrak{A}_{p}$ in $I_{K, P}=K_{p}^{*}$

$$
N_{K_{p} / k_{p}} \mathfrak{A}_{p}=N_{K / k} \mathfrak{A}_{p} \text {. }
$$

Let $L_{p}=k_{p} v^{*}(L)$ with $v$ in $\not \cap N, B_{p}$ the maximum abelian extension of $k_{p}$ in $L_{p}, L_{p}^{*}$ and $B_{p}^{*}$ the multiplicative groups of the nonzero elements in $L_{p}$ and $B_{p}$, respectively. Then, $N_{B_{p} / k_{p}} B_{p}^{*}=N_{L_{p} / k_{p}} L_{p}^{*}$. As $K_{P} \cap H(L / K)=N_{L_{p} / K_{p}} L_{p}^{*}$, the intersection $F_{P}^{*}=F^{*} \cap K_{P}\left(=F^{*} \cap I_{K, P}\right)$ contains the inverse image in $K_{p}$ by $N_{K_{p} / k_{p}}$ of $N_{B_{p} / k_{p}} B_{p}^{*}$, so, as $L^{\prime} / k$

'Cf. [5], esp. Lemma 3. 1.c. 
is Galois, $L^{\prime}$ is EL-abelian over $K / k$. Let $E^{*}$ be the subset of $I_{K}$ consisting of all of such idèles $\mathfrak{A}$ which can be written as $\mathfrak{A}=\mathfrak{B}^{1-s}$ with $\mathfrak{B}$ in $I_{K}$ and $s$ in $G(K / k)$, and $E^{*}$ is contained in $F^{*}$, so $G\left(L^{\prime} / K\right)$ lies in the center of $G\left(L^{\prime} / k\right)$. Therefore, $L^{\prime}$ is contained in $D$ and we obtain, from (7), $F=F^{*}$, which we state as the following theorem.

Theorem 2. Let $K / k$ be finite, separably Galois extension, L a finite, separably algebraic extension of $K$, Galois over $k$ and abelian over $K$, and $D$ be the maximum extension of $K$ in $L$, such that $D / K$ is central (in the sense stated in the preface) and EL-abelian over $K / k$. Then, the idele group $H(D / K)$ is the inverse image in $I_{K}$ by $N_{K / k}$ of $N_{K / k} H(L / K)$.

From Theorem 1 and Theorem 2, it follows evidently a formal generalization of the isomorphism theorem in the class field theory, which we state as the following theorem.

THEOREM 3. $\sigma_{K / k}$ gives canonically a homomorphism $\sigma_{K / k}^{D}$ of $N_{K / k} I_{K}$ onto $G(D / K)$, having $N_{K / k} P_{K} \cdot N_{L / k} I_{L}$ as its kernel, where we denote by $P_{K}$ the principal idele group of $K$.

Applying Theorem 3 to the restriction of $\sigma_{K / k}$ into $N_{K / k} I_{K, P}$, we obtain from the local class field theory the following decomposition theorem.

Theorem 4. Let $D_{p}=k_{p} v^{*}(D)$ with $v$ in $\bar{p} \cap N$ and $D_{p}^{*}$ be the multiplicative group of the nonzero elements in $D_{p}$. Then, $N_{D_{p} / k_{p}} D_{p}^{*}=k_{p}$ $\cap\left(N_{K / k} P_{K} \cdot N_{L / k} I_{L}\right)$.

We obtain from Theorem 2 and Theorem 3 the kernel of $\sigma_{K / k}$, as follows.

TheOREM 5. $\sigma_{K / k}$ is a homomorphism of $N_{K / k} I_{K}$ onto a dense subgroup of $G\left(D_{K / k} / K\right)$ with the Krull topology and the kernel consists of the ideles belonging to elements of the closure of the unit element of $N_{K / k} I_{K} / N_{K / k} \Delta_{K}$ with the Krull topology, ${ }^{6}$ where we denote by $\Delta_{K}$ the subgroup of $I_{K}$ consisting of the ideles belonging to elements of the connected component of the unit element of the idele class group $I_{K} / P_{K}$.

Proof. Let $L^{\prime}$ be a subgroup of $N_{K / k} I_{K}$, containing $N_{K / k} \Delta_{K}$ such that $\left[N_{K / k} I_{K}: L^{\prime}\right]$ is finite. Evidently, the inverse image in $I_{K}$ of $L^{\prime}$ by $N_{K / k}$ corresponds to a class field $L / K$ which is of finite degree and separably Galois over $k$. Then, it follows from Theorem 2, $L=L \cap D_{K / k}$. Then, the kernel of $\sigma_{K / k}^{L}$ is, from Theorem $3, L^{\prime}$, and the kernel of

${ }^{6}$ Let $U$ be a group. We call the topology of $U$ having as neighborhoods of the unit element subgroups $V$ such that $[U: V]$ is finite the Krull topology of $U$, which is not necessarily Hausdorff. 
$\sigma_{K / k}$ is the intersection of all of such $L^{\prime}$, which is the last half of the theorem. The rest of the theorem is clear, q.e.d.

From now on, we shall study, in $\S 3$, the nonabelian part in the extension $D_{K / k} / k$, and obtain our main result which concerns total norm residues in the principal idèle group $P_{k}$ of $k$ for $K / k$.

3. $P_{k} \cap N_{K / k} I_{K}$. Let $L$ be a finite separable extension of $K$ in $\Omega$ such that $L / k$ is Galois and $L / K$ is abelian. Let $A, B, C, D$, and $\sigma_{K / k}^{D}$ be as stated in $\S 2$. As the inverse image in $I_{K}$ by $N_{K / k}$ of $H(A K / K)$ is $H(A K / K)$ itself, it follows from Theorem 1 and Theorem 3 that $\sigma_{K / k}^{D}\left(N_{K / k} \mathfrak{A}\right)$ belongs to $G(D / A K)$ for $\mathfrak{A}$ in $I_{K}$, if and only if $\mathfrak{A}$ belongs to $H(A K / K)$, where we denote by $H(A K / K)$ the subgroup of $I_{K}$ corresponding to $A K / K$. Then, we obtain evidently from Theorem 3 the following theorem.

Theorem 6'. The inverse image of $G(D / A K)$ by $\sigma_{K / k}^{D}$ is $H(A / k)$ $\cap N_{K / k} I_{K}$ and it holds $G(D / A K) \cong\left(H(A / k) \cap N_{K / k} I_{K}\right) / N_{L / k} H(L / K)$, where we denote by $H(L / K)$ the subgroup of $I_{K}$ corresponding to $L / K$.

As to $G\left(D_{K / k} / A_{k} K\right)$, it holds the following theorem.

Theorem 6. $\sigma_{K / k}(\mathfrak{a})$ belongs to $G\left(D_{K / k} / A_{k} K\right)$ for $\mathfrak{a}$ in $N_{K / k} I_{K}$, if and only if a belongs to $\Delta_{k} \cap N_{K / k} I_{K}$ and $\sigma_{K / k}$ gives canonically a homomorphism of $\left(\Delta_{k} \cap N_{K / k} I_{K}\right) / N_{K / k} \Delta_{K}$ onto $G\left(D_{K / k} / A_{k} K\right)$, where we denote by $\Delta_{k}$ and $\Delta_{K}$ the subgroups of $I_{k}$ and $I_{K}$ consisting of the ideles belonging to elements of the connected components of the unit elements of $I_{k} / P_{k}$ and $I_{K} / P_{K}$, respectively.

Proof. The first half of the theorem follows, as the transition theorem in the class field theory holds for the infinite extension $A_{k} / K$ in the same manner as for the finite extension $A / K$, from Theorem 1 and Theorem 5 in place of Theorem 3 , in the same manner as Theorem $6^{\prime}$. The rest of the theorem is evident, if $k$ is algebraic number field. Let $k$ be algebraic function field. $A_{k}$ contains, then, an algebraic closure of the constant field of $K$, and the norm residue symbol $\sigma_{K}^{\prime}$ for $D_{K / k} / K$ gives canonically a homomorphism of a subgroup of $I_{K}$ onto $G\left(D_{K / k} / A_{k} K\right)$, so, $\sigma_{K / k}$ is, from Theorem 1 , an onto homomorphism, q.e.d.

As, from the supposition $K / k$ is of finite degree, it holds, as is well known,

$$
P_{k} N_{K / k} I_{K}=\Delta_{k} N_{K / k} I_{K} .
$$

We obtain from the natural homomorphism of $\Delta_{k}$ onto

$$
\left(\Delta_{k} N_{K / k} I_{K}\right) / N_{K / k} I_{K}=\left(P_{k} N_{K / k} I_{K}\right) / N_{K / k} I_{K} \cong P_{k} /\left(P_{k} \cap N_{K / k} I_{K}\right)
$$


a homomorphism of $\Delta_{k}$ onto $P_{k} /\left(P_{k} \cap N_{K / k} I_{K}\right)$ which has $\Delta_{k} \cap N_{K / k} I_{K}$ as its kernel and coincides, if restricted into $P_{k}$, with the natural mapping of $P_{k}$ onto $P_{k} /\left(P_{k} \cap N_{K / k} I_{K}\right)$. Thus, we obtain the following theorem.

THEOREM 7. $\Delta_{k} /\left(P_{k} \cap N_{K / k} I_{K}\right)=\left(\Delta_{k} \cap N_{K / k} I_{K}\right) /\left(P_{k} \cap N_{K / k} I_{K}\right)$ $\times P_{k} /\left(P_{k} \cap N_{K / k} I_{K}\right)$, where $\times$ denotes direct product.

Let $H_{k}^{\prime}$ and $H_{K}^{\prime}$ be the maximal compact subgroups in the connected components of the unit elements of $I_{k}$ and $I_{K}$, respectively. As is well known, $H_{k}^{\prime}=N_{K / k} H_{K}^{\prime}, H_{k}^{\prime} \cap P_{k}=1$, and $H_{k}^{\prime} \subset\left(\Delta_{k} \cap N_{K / k} I_{K}\right)$, so we obtain

$$
P_{k}^{\prime} \cap N_{K / k} I_{K}=H_{k}^{\prime} \times\left(P_{k} \cap N_{K / k} I_{K}\right),
$$

where we denote $P_{k} H_{k}^{\prime}$ by $P_{k}^{\prime}$. Thus, we obtain from Theorem 7 the following lemma.

Lemma 1. $\left(\Delta_{k} \cap N_{K / k} I_{K}\right) /\left(P_{k}^{\prime} \cap N_{K / k} I_{K}\right) \cong \Delta_{k} / P_{k}^{\prime}$.

Let $[K: k]=n$, and we shall prove the following lemma.

Lemma 2.

$$
\begin{gathered}
\left(\Delta_{k} \cap N_{K / k} I_{K}\right) /\left(P_{k}^{\prime} \cap N_{K / k} I_{K}\right)^{n} \\
=\left(P_{k}^{\prime} \cap N_{K / k} I_{K}\right) /\left(P_{k}^{\prime} \cap N_{K / k} I_{K}\right)^{n} \times\left(\left(\Delta_{k} \cap N_{K / k} I_{K}\right) /\left(P_{k} \cap N_{K / k} I_{K}\right)\right)^{n} .
\end{gathered}
$$

Proof. Let $a \in \Delta_{k} \cap N_{K / k} I_{K} .\left(\Delta_{k} \cap N_{K / k} I_{K}\right) /\left(P_{k}^{\prime} \cap N_{K / k} I_{K}\right)$ is, from Lemma 1 , divisible and uniquely divisible, ${ }^{7}$ and there exists $\mathfrak{x} \in \Delta_{k}$ $\cap N_{K / k} I_{K}$ and $\alpha \in P_{k}^{\prime} \cap N_{K / k} I_{K}$ such that $\mathfrak{a}=\mathfrak{x}^{n} \alpha$. Such $\alpha$ is clearly uniquely determined by $\mathfrak{a}$ up to factors in $\left(P_{k}^{\prime} \cap N_{K / k} I_{K}\right)^{n}$, so, we obtain a homomorphism of $\left(\Delta_{k} \cap N_{K / k} I_{K}\right) /\left(P_{k}^{\prime} \cap N_{K / k} I_{K}\right)^{n}$ onto $\left(P_{k}^{\prime} \cap N_{K / k} I_{K}\right) /\left(P_{k}^{\prime} \cap N_{K / k} I_{K}\right)^{n}$ which has

$$
\left(\left(\Delta_{k} \cap N_{K / k} I_{K}\right) /\left(P_{k}^{\prime} \cap N_{K / k} I_{K}\right)\right)^{n}
$$

as its kernel, coincides, if restricted into $\left(P_{k}^{\prime} \cap N_{K / k} I_{K}\right) /\left(P_{k}^{\prime} \cap N_{K / k} I_{K}\right)^{n}$, with the identity, which certifies evidently the lemma, q.e.d.

As $\left(P_{k}^{\prime} \cap N_{K / k} I_{K}\right)^{n} \subset N_{K / k} P_{K}^{\prime} \subset P_{k}^{\prime} \cap N_{K / k} I_{K}$, the following corollary follows from Lemma 2 .

CoRollary. $\quad\left(\Delta_{k} \cap N_{K / k} I_{K}\right) / N_{K / k} P_{K}^{\prime}=\left(P_{k}^{\prime} \cap N_{K / k} I_{K}\right) / N_{K / k} P_{K}^{\prime}$ $\times\left(\left(\Delta_{k} \cap N_{K / k} I_{K}\right) /\left(P_{k}^{\prime} \cap N_{K / k} I_{K}\right)\right)^{n}$.

As, from Lemma $1,\left(\left(\Delta_{k} \cap N_{K / k} I_{K}\right) /\left(P_{k}^{\prime} \cap N_{K / k} I_{K}\right)\right)^{n}$ is divisible and uniquely divisible, $\sigma_{K / k}$ maps idèles belonging to elements in it onto the unit element of $G\left(D_{K / k} / A_{k} K\right)$. Then, it follows from Lemma 2

${ }^{7}$ Cf. [4]. 
and Theorem 6 that $\sigma_{K / k}$ maps $P_{k}^{\prime} \cap N_{K / k} I_{K}$ onto $G\left(D_{K / k} / K A_{k}\right)$ and the kernel contains $N_{K / k} P_{K}{ }^{\prime}$. As, obviously,

$$
\left(P_{k} \cap N_{K / k} I_{K}\right) / N_{K / k} P_{K}^{\prime}=\left(\left(P_{k} \cap N_{K / k} I_{K}\right) \times H_{k}^{\prime}\right) / H_{k}^{\prime} N_{K / k} P_{K},
$$

it holds

$$
\sigma_{K / k}\left(P_{k} \cap N_{K / k} I_{K}\right)=G\left(D_{K / k} / A_{k} K\right) .
$$

In the following, we shall prove that the kernel of $\sigma_{K / k}$ restricted into $P_{k} \cap N_{K / k} I_{K}$ is $N_{K / k} P_{K}$. For that purpose, we prepare the following lemma, which is a modification of Theorem 5 .

Lemma 3. The kernel of $\sigma_{K / k}$ consists of ideles belonging to elements of the closure of the unit element of $N_{K / k} I_{K} / N_{K / k} P_{K}^{\prime}$ with the Krull topology.

Proof. Let $L^{\prime}$ be a subgroup of $N_{K / k} I_{K}$, containing $N_{K / k} P_{K}^{\prime}$ such that $\left[N_{K / k} I_{K}: L^{\prime}\right]$ is finite. As $\Delta_{K} / P_{K}^{\prime}$ is divisible, so is $N_{K / k} \Delta_{K} / N_{K / k} P_{K}^{\prime}$, and $L^{\prime}$ contains $N_{K / k} \Delta_{K}$. Then, it follows evidently from Theorem 5 the lemma, q.e.d.

Decomposing $\left(\Delta_{k} \cap N_{K / k} I_{K}\right) / N_{K / k} P_{K}^{\prime}$ as direct product, as stated in corollary to Lemma 2, we obtain, from Lemma 3 , as the latter factor is a divisible group, that the kernel of $\sigma_{K / k}$ restricted into $P_{k}^{\prime} \cap N_{K / k} I_{K}$ is the subgroup consisting of the idèles belonging to elements of the closure of the unit element of $\left(P_{k}^{\prime} \cap N_{K / k} I_{K}\right) / N_{K / k} P_{K}^{\prime}$ with the Krull topology. Then, we obtain, in the same manner as in the proof of (11), the following lemma.

Lemma 4. The kernel of $\sigma_{K / k}$ restricted into $P_{k} \cap N_{K / k} I_{K}$ is the subgroup $W$ consisting of the ideles belonging to elements of the closure of the unit element of $\left(P_{k} \cap N_{K / k} I_{K}\right) / N_{K / k} P_{K}$ with the Krull topology.

$\left(P_{k} \cap N_{K / k} I_{K}\right) / N_{K / k} P_{K}$ has, as abelian discrete group, sufficiently many representations, which are, as every element of

$$
\left(P_{k} \cap N_{K / k} I_{K}\right) / N_{K / k} P_{K}
$$

has an order dividing $n$ homomorphisms into a cyclic group of order $n$, so, the closure of the unit element of $\left(P_{k} \cap N_{K / k} I_{K}\right) / N_{K / k} P_{K}$ with the Krull topology consists only of the unit element. Thus, we obtain the following theorem, which is the main result of the present paper.

THEOREM 8. $\sigma_{K / k}$ gives canonically an isomorphism of

$$
\left(P_{k} \cap N_{K / k} I_{K}\right) / N_{K / k} P_{K} \text { onto } G\left(D_{K / k} / A_{k} K\right) \text {. }
$$

The author is greatly indebted to the referees for many suggestions for improving the paper. 


\section{REFERENCES}

1. C. Chevalley, Sur la théorie du corps de classes dans les corps finis et corps locaux, Journ. of Coll. of Sciences, Tokyo, II, vol. 9 (1933).

2. - La theorie du corps de classes, Ann. of Math. no. 2 vol. 41 (1940).

3. A. Scholz, Totale Normenreste, die keine Normen sind, als Erzeuger nichtabelscher Körpererweiterungen, no. 2, Crelle J. vol. 182 (1940).

4. A. Weil, Sur la théorie du corps de classes, J. Math. Soc. Japan vol. 3 (1951).

5. G. Whaples, Non-analytic class field theory and Grunwald's theorem, Duke Math. J. vol. 9 (1942).

YAMAGATA, JAPAN

\section{ON SMOOTH LINEAR PARTIAL DIFFERENTIAL EQUATIONS WITHOUT SOLUTIONS ${ }^{1}$}

\section{PHILIP HARTMAN}

1. H. Lewy [4] has recently constructed a system of first order, linear partial differential equations with coefficients of class $C^{\infty}$ having the property that it possesses no "solution" on any domain. By "solution" is meant a solution having uniformly Hölder continuous partial derivatives. Since the "Hölder continuity" requirement on the partial derivatives is rather artificial, there arises the question as to whether or not there is such a system which possesses no $C^{1}$ (or even no weak $\left.L^{2}\right)$ solution on any domain. The object of this note is to answer this question in the affirmative. The desired system will be obtained by a modification of Lewy's example.

Lewy's system involves two (real) unknown functions $\left(u^{1}, u^{2}\right)$ and three (real) independent variables $(s, x, y)$. On putting $u=u^{1}+i u^{2}$, Lewy writes his example in the form

$$
L(u)=F(s, x, y),
$$

where $L$ is the homogeneous, linear, first order, partial differential operator with analytic coefficients,

Received by the editors July 30, 1958.

1 The preparation of this paper was sponsored by the Office of Naval Research. Reproduction in whole or in part is permitted for any purpose of the United States Government. 